Алгебра и анализ

Том 20 (2008), № 5
St. Petersburg Math. J.

Vol. 20 (2009), No. 5, Pages 687-707

S 1061-0022(09)01068-1

Article electronically published on July 21, 2009

\title{
NUMEROLOGY OF SQUARE EQUATIONS
}

\author{
N. A. VAVILOV
}

\begin{abstract}
In the present work, which is a sequel of the paper "Can one see the signs of structure constants?", we describe how one can see the form and the signs of the senior Weyl orbit of equations on the highest weight orbit directly in the weight diagram of microweight representations and adjoint representations for the simplylaced case. As special cases, the square equations we consider include the vanishing of second order minors, Plücker equations in polyvector and adjoint representations of classical groups, Cartan equations in spin and half-spin representations, BorelFreudenthal equations defining the projective octave plane $\mathrm{E}_{6} / P_{1}$, and most of the equations defining Freudenthal's variety $\mathrm{E}_{7} / P_{7}$. In view of forthcoming applications to the construction of decomposition of unipotents in the adjoint case, special emphasis is placed on the senior Weyl orbit of equations for the adjoint representations of groups of types $\mathrm{E}_{6}, \mathrm{E}_{7}$, and $\mathrm{E}_{8}$. This orbit consists of 270,756 , or 2160 equations, respectively, and we minutely discuss their form and signs. This generalizes Theorem 3 of the preceding paper "A third look at weight diagrams", where we considered microweight representations of $\mathrm{E}_{6}$ and $\mathrm{E}_{7}$.
\end{abstract}

Reporting the work on decomposition of unipotents, [11, 45, 46, 47, 50, at the Darstellungstheorieseminar in Bielefeld, I have drawn the following picture:

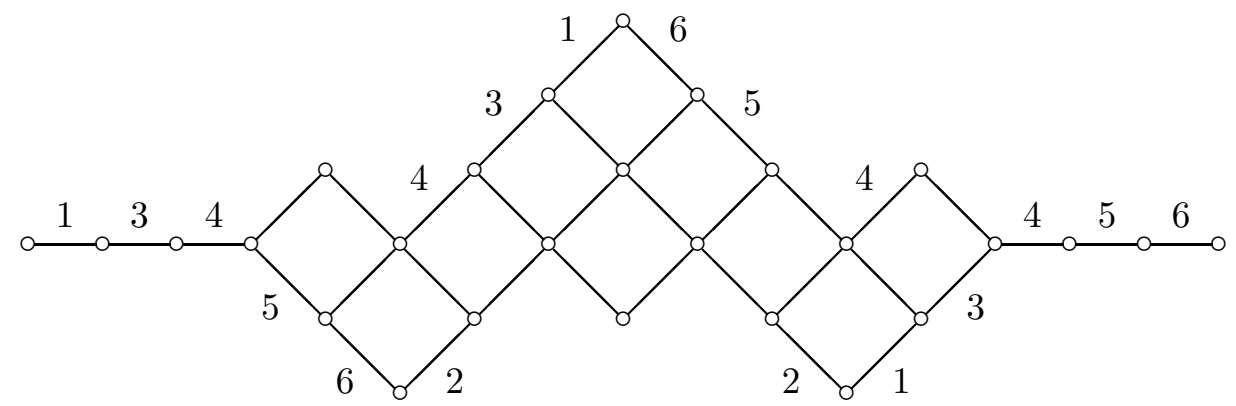

FiguRe 1. $\left(\mathrm{E}_{6}, \varpi_{1}\right)$

2000 Mathematics Subject Classification. Primary 20G15, 20G35.

Key words and phrases. Chevalley groups, polyvector representations, spin representations, minimal modules, highest weight orbit, weight diagram, crystal graph, standard monomial theory, decomposition of unipotents, Plücker equations, Cartan equations, Borel-Freudenthal equations, projective octave plane.

The basic ideas, which eventually led to the present paper, as also to 47 4] - a third look - were developed by the author in 1995 at the Universität Bielefeld (with the support of AvH-Stiftung, SFB-343 and INTAS 93-436). Preliminary versions of this text were taking shape since 1997, at the Universitá Milano I (with the support of the Cariplo Foundation for Fundamental Research), at the Newton Institute for Mathematical Sciences at Cambridge University, and at Bar Ilan University. At the final stage of this work the author was supported by RFBR 03-01-00349 (POMI RAN), by INTAS 00-566, and INTAS 03-51-3251, and by an express grant of the Russian Ministry of Education 'Overgroups of semi-simple groups' E02-1.0-61. 
Claus Ringel immediately asked, whether there is any relationship between the two following facts: there are exactly 10 out of 27 equations defining the highest weight orbit, in the representation $\left(\mathrm{E}_{6}, \varpi_{1}\right)$, that reduce dimension, whereas other equations cut out connected components; there are exactly 10 squares in this picture? This is indeed the case, and the resulting answer is called the theory of standard monomials [43, 30, 31, 32, 33, 34, 35. Actually, the number of squares in this picture equals 27, and each of them personifies a unique square equation. The 10 small squares, those that catch one's eye, correspond to independent equations, defining a complete intersection.

In the present paper I explain how to restore the shape and the signs of square equations directly from the weight diagram of a representation, without any reference to lofty geometric doctrines 1 It is well known [36] that the resulting equations have degree 2; in other words, square equations are indeed quadratic.

Thus, the present paper is a sequel of [4] in which I systematically continue to generalize results of [47] to all microweight representations, and to adjoint representations of types $\mathrm{A}_{l}, \mathrm{D}_{l}, \mathrm{E}_{l}$. In [4] I generalized Theorems 1 and 2 of [47] to this situation; these theorems pertain to the action structure constants. In the present paper I essentially obtain a similar generalization of Theorem 3 thereof, pertaining to equations. As special instances, the kinds of equations we consider here include the following examples of square equations.

- Vanishing of second order minors in the adjoint representation, and in tensor products of vector/covector representations of Chevalley groups of type $\mathrm{A}_{l}$.

- Plücker equations in polyvector representations of Chevalley groups of type $\mathrm{A}_{l}$ and in the adjoint representation of Chevalley groups of type $\mathrm{D}_{l}$.

- Cartan equations in spin and half-spin representations of Chevalley groups of type $\mathrm{D}_{l}$.

- Borel-Freudenthal equations, which define the projective octonion plane $\mathrm{E}_{6} / P_{1}$. These equations are obtained by equating to zero the first order partial derivatives of the invariant cubic form [16, 17, 20] in the 27-dimensional representation of the Chevalley group of type $\mathrm{E}_{6}$.

- Most of Freudenthal equations, defining the Freudenthal variety $\mathrm{E}_{7} / P_{7}$. These equations are obtained by equating to zero some second order partial derivatives of the invariant quartic form [17, 24, 29], in the 56-dimensional representation of the Chevalley group of type $\mathrm{E}_{7}$.

- The Weyl orbit of the highest equation defining the highest weight orbit, for the adjoint representations of Chevalley groups of types $\mathrm{E}_{6}, \mathrm{E}_{7}$ and $\mathrm{E}_{8}$. This orbit consists of 270,756 , or 2160 equations, respectively.

In general, these equations do not exhaust all equations defining the highest weight orbit. Nevertheless, it turns out that in the last example, these equations, together with exactly one additional quadratic equation suffice to construct decomposition of unipotents in adjoint representations of Chevalley groups of types $\mathrm{E}_{6}, \mathrm{E}_{7}$, and $\mathrm{E}_{8}$. This extra equation follows from the fact that the image of the adjoint representation is contained in the orthogonal group.

In a forthcoming paper [5], I intend to generalize the principal results of [47, Theorems 4 and 5 , to the adjoint representation of Chevalley groups of type $\mathrm{E}_{6}$. This shall finalize an a priori proof of the decomposition of unipotents for this case. Unlike our original proofs sketched in [11, 46, 50, this new proof is computer independent. Unfortunately, as in 47, to do this we have to scrutinize signs of the structure constants and equations, and this is exactly what we do in 4 . and the present paper. I have no doubt

\footnotetext{
${ }^{1}$ Both those pertaining to algebraic geometry, and those pertaining to combinatorial geometry!
} 
that a similar proof should work also for the cases $\mathrm{E}_{7}$ and $\mathrm{E}_{8}$. On the other hand, one can expect that they will be significantly harder from a technical viewpoint. As a matter of fact, in [5], we can profit from additional symmetries stemming from the embedding $\mathrm{D}_{4} \subseteq \mathrm{E}_{6}$.

The author dedicates this series of papers to the memory of Dmitry Konstantinovich Faddeev. I have not been his direct apprentice, but for me, as for all algebraists of my generation in Saint-Petersburg, he was more than a God, he was a Teacher.

\section{$\S 1$. INTRODUCTION}

We keep the notation used in [4] and [6]; see also [42, 46, 47, 50] and the references therein. Let $\Phi$ be a reduced irreducible root system of rank $l$. We are basically interested in the case where all roots $\Phi$ have the same length. Such root systems are usually called simply-laced, as opposed to multiply-laced systems. Next, let $\Pi=\left\{\alpha_{1}, \ldots, \alpha_{l}\right\}$ be a fundamental system in $\Phi$ (its elements will be called fundamental roots), let $\Phi^{+}$ and $\Phi^{-}$be the corresponding sets of positive and negative roots, respectively, and let $Q(\Phi)=\mathbb{Z} \alpha_{1} \oplus \cdots \oplus \mathbb{Z} \alpha_{l}$ be the corresponding root lattice. We always use the same fundamental roots numbering as in 2. As always, $W=W(\Phi)$ denotes the Weyl group of $\Phi ; w_{\alpha}$ is the reflection relative to the root $\alpha \in \Phi$, and $s_{i}=w_{\alpha_{i}}, 1 \leq i \leq l$, denotes fundamental reflections. By $l(w)$ we denote the length of $w \in W$ with respect to Coxeter generators $s_{1}, \ldots, s_{l}$.

Further, let $G=G(\Phi, R)$ be the simply connected Chevalley group of type $\Phi$ over a commutative ring $R$ with 1 . All necessary general definitions can be found, for instance, in 1, 15, 18, whereas in the papers [46, 47, 50, one can find many further references related to Chevalley groups over rings. We fix a split maximal torus $T=T(\Phi, R)$ in $G$. As usual, $X_{\alpha}=\left\{x_{\alpha}(\xi), \xi \in R\right\}, \alpha \in \Phi$, denotes a unipotent root subgroup in $G$, elementary with respect to $T$. For $\varepsilon \in R^{*}$ the element $w_{\alpha}(\varepsilon)$ is defined as $w_{\alpha}(\varepsilon)=x_{\alpha}(\varepsilon) x_{-\alpha}\left(-\varepsilon^{-1}\right) x_{\alpha}(\varepsilon)$.

As usual, $P(\Phi)$ is the lattice of integral weights of $\Phi$, while $P_{++}(\Phi)$ denotes the cone of dominant integral weights. Recall that every weight $\omega \in P_{++}(\Phi)$ is a nonnegative integer linear combination of the fundamental weights $\varpi_{1}, \ldots, \varpi_{l}$. We fix a dominant weight $\omega \in P_{++}(\Phi)$ and let $V=V(\omega)$ be the Weyl module of the group $G$ with the highest weight $\omega$. The corresponding representation $G \longrightarrow \mathrm{GL}(V)$ will be denoted by $\pi=\pi(\omega)$. By $\Lambda(\pi)=\Lambda(\omega)$ we denote the set of weights of the representation $\pi$ with multiplicities. One can choose an admissible base $v^{\lambda}, \lambda \in \Lambda(\omega)$, of $V$, consisting of weight vectors. In other words, every $v^{\lambda}$ is indeed a vector of weight $\lambda$, viewed as a weight in the usual sense, without multiplicities. The action of root unipotents $x_{\alpha}(\xi), \alpha \in \Phi, \xi \in R$, in this base is expressed by matrices whose entries are polynomials in $\xi$ with integer coefficients.

As in 47, 6, 4, our main tools are weight diagrams, and we refer an interested reader to [37, 42, 44, 46, 47, where one can find a description of their use in the theory of Chevalley groups and a few dozen further references. In the first approximation, the weight diagram of a representation $\pi$ is a marked oriented graph or, in the terminology of Kashiwara, a colour graph. The vertices correspond to the weights of the representation $\pi$, usually with multiplicities. Two vertices $\lambda$ and $\mu$ are joined by an arrow with label $i$ ( $=$ of colour $i$ ), directed from $\mu$ to $\lambda$ if $\lambda-\mu=\alpha_{i}$ is the $i$ th fundamental root.

In the present paper we are basically interested in the following two simplest special cases:

- microweight representations, where all weights are extremal;

- adjoint representations for simply laced root systems, where a unique nonextremal weight is the zero weight. 
In the first of these cases, the multiplicity problem does not occur at all, since all weights have multiplicity 1 . Thus, for a microweight representation the weight diagram, which in this case is isomorphic to the coset adjacency diagram of the Weyl group $\Phi$, modulo the stabilizer of the highest weight, coincides with the crystal graph of Kashiwara. Let us sketch a solution for the adjoint representations, which also works for multiply-laced systems. The set of weights $\Lambda(\omega)$ is the union of the set $\Lambda^{*}(\omega)=\Phi$ of nonzero weights and the set consisting of zero weights $\widehat{\alpha}_{1}, \ldots, \widehat{\alpha}_{l}$, indexed by the fundamental roots. A zero vertex $\widehat{\alpha}_{i}$ is only joined to $\alpha_{i}$ and to $-\alpha_{i}$. As was proved by Robert Marsh, this construction again leads to the crystal graph.

In 4] we reproduced some most interesting weight diagrams, for the cases of $\mathrm{E}_{6}, \mathrm{E}_{7}$, and $\mathrm{E}_{8}$. The diagrams of all microweight and adjoint representations, as also representations on short roots, are collected in 42. These diagrams appear in various branches of mathematics, starting with representation theory itself, the theory of algebraic groups and invariant theory, up to algebraic $K$-theory, algebraic and differential geometry, combinatorics, finite geometries, the theory of nonassociative algebras, probability theory, mathematical physics, etc. A detailed discussion of weight diagrams and their uses, and also a number of further references, can be found in [4, 8, 37, 38, 40, 41, 42, 44, 46, 47, 50].

Originally, weight diagrams occurred as a purely combinatorial object that describes the Bruhat order or Duflo order on cosets of the Weyl group $W=W(\Phi)$ modulo a parabolic subgroup $W_{J}=\left\langle s_{j}, j \in J\right\rangle$, for some $J \subseteq \Pi=$ first look. The vertices of such a diagram correspond exclusively to the Weyl orbit of the highest weight. Since all such weights are extremal, the multiplicity problem does not occur at all.

Soon thereafter it was realized that in fact weight diagrams describe the action of a Lie group/algebra up to signs = second look; see [37, 44]. Namely, vectors in $V=V(\omega)$ can be visualized as follows. Let us fix an admissible base $v^{\lambda}, \lambda \in \Lambda(\omega)$ in $V(\omega)$. Then a vector

$$
a=\sum a_{\lambda} v^{\lambda} \in V, \quad a_{\lambda} \in R, \quad \lambda \in \Lambda(\omega)
$$

(in the sequel written simply as $a=\left(a_{\lambda}\right)$ ) will be depicted by a marked graph obtained from the weight diagram $(\Phi, \omega)$ by inserting $a_{\lambda}$ in the vertex that corresponds to $\lambda$. Now a root unipotent $x_{\alpha_{i}}(\xi), \alpha_{i} \in \pm \Pi, \xi \in R$, corresponding to a positive/negative fundamental root, acts along the edges of the diagram with label $i$ in the positive/negative direction. For an arbitrary root $\alpha \in \Phi$ the action of $x_{\alpha}(\xi)$ is described by directed paths with labels determined by the expansion of $\alpha$ in fundamental roots, in an arbitrary order. See [42, 46, 47, 50, for the details.

Finally, in the 1990s it became clear that weight diagrams describe action completely, including signs $=$ third look. As a matter of fact, the crystal bases possess remarkable positivity properties. Using these properties one can completely restore the action by its diagram. For the cases of $\left(\mathrm{E}_{6}, \varpi_{1}\right)$ and $\left(\mathrm{E}_{7}, \varpi_{7}\right)$, an algorithm and an elementary proof that uses realization of these modules in the unipotent radicals of parabolic subgroups were produced in [47. In 4 we expounded the corresponding algorithms and direct proofs for all microweight modules, and for adjoint modules of type $\mathrm{A}_{l}, \mathrm{D}_{l}$, and $\mathrm{E}_{l}$.

In the present paper we pursue this line and show how to see equations that define the highest weight orbit directly in the weight diagram. For a moment, we assume that $R=K$ is an algebraically closed field, and that $G$ is viewed as an algebraic group. In this case it is well known that in any representation the closure of the highest weight orbit is an intersection of quadrics [36]. For the representations considered in the present paper the precise shape of these quadratic equations is predicted by the theory of standard monomials; see [43, 30, 31, 32, 33, 34, 35. The simplest and most important equations on a column $x=\left(x_{\lambda}\right), \lambda \in \Lambda(\pi)$, have the form

$$
x_{\lambda_{1}} x_{\mu_{1}} \pm x_{\lambda_{2}} x_{\mu_{2}} \pm \cdots \pm x_{\lambda_{m}} x_{\mu_{m}}=0
$$


for some weights $\lambda_{1}, \ldots, \lambda_{m}, \mu_{m}, \ldots, \mu_{1}$ forming a diagram of type $\left(\mathrm{D}_{m}, \varpi_{1}\right)$ reproduced in Figure 2.

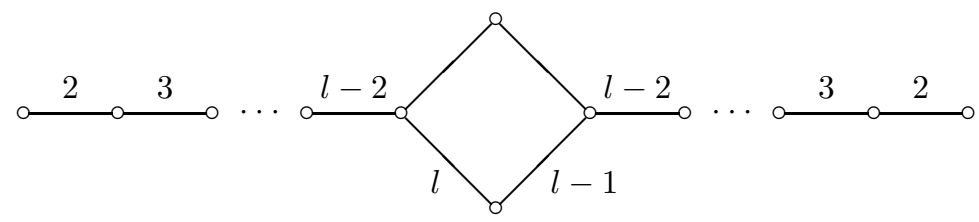

FIGURE 2. $\left(\mathrm{D}_{l}, \bar{\omega}_{1}\right)$

In $\S \S 2-5,7$, and 8 we discuss how to specify signs in these equations, and how to see them in the diagram (Theorems 1-3). Of course, for a ring $R$, a vector subject to these equations may fail to be the first column of a matrix $g \in G$. There are obstacles of a completely different nature (the $\mathrm{K}_{0}$-functor and its analogs). However, in any case these equations represent necessary conditions and play a central role in many calculations.

It seems to us that results of the present paper are of independent interest. Together with the results of [4, they furnish a completely elementary approach to calculations in exceptional groups, accessible to an undergraduate student. Nevertheless, there is a specific application we have in mind, namely, an a priori proof for decomposition of unipotents in the corresponding representations. Namely, in [11, we proposed a new approach towards the proof of the main structure theorems for Chevalley groups over a commutative ring, based on the geometry of minimal modules. Roughly, the idea of this approach can be stated as follows. All calculations necessary to prove the main structure theorems can be organized so as to use only the following: elementary calculations, based on Steinberg relations, and calculations involving one column or one row of a matrix at a time. For reasons we cannot discuss here, in [1] they were called stable calculations. A specific method to reduce general calculations to elementary and stable ones, proposed in [11, is called decomposition of unipotents.

For classical groups in vector representation, it is easy to carry out the program outlined in [11. Thereby one does not encounter major technical complications, even in much more general situations; see in particular [45, 46] and the references therein. Yet, taking care of all technical details for exceptional groups turned out to be much more arduous than it seemed at the early stages of the work. To check that signs of the action constants comply with equations, we had to rely on extensive computer calculations. The outline of the proof in [46 omits such a verification. Finally, in 47, we succeeded in giving a human proof for decomposition of unipotents in microweight representations for groups of types $\mathrm{E}_{6}$ and $\mathrm{E}_{7}$. The results of the present paper constitute roughly one-fourth of a similar a priori proof in adjoint representations for groups of types $\mathrm{E}_{6}, \mathrm{E}_{7}$, and $\mathrm{E}_{8}$. Other parts of the proof consist in a geometric analysis of equations defining the sheet containing the highest weight orbit, in the study of root type elements (addition and commutation formulas, an analog of the Whitehead lemma, etc.), and in stabilization of a column and the proof of decomposition of unipotents. We intend to do this in subsequent publications; see [46, 50] for a description of the whole project.

The paper is organised as follows. In $\S 2$ we state basic results on the shape of square equations, Theorems 1 and 2 , and in $\S \S 3-5$ we prove these results. In $\S 6$ we discuss some exceptional numerology pertaining to adjoint representations for groups of types $\mathrm{E}_{6}, \mathrm{E}_{7}$, and $\mathrm{E}_{8}$. In $\S 7$ we prove another main result of the paper, Theorem 3, that specifies signs of monomials in square equations. Finally, in $\S 8$ we describe how to find square equations in the diagram. 


\section{$\S 2$. SQUARES}

Eventually, I started to classify. And my classification is simple beyond comparison.

Mikhail Bulgakov, Moscow of the 1920s

Now we pass to the main topic of the present paper. We consider one remarkable type of equations satisfied by the orbit of the highest weight vector. We do not try to delve into profound geometric aspects, and we carry out a purely combinatorial analysis of the shape of these equations and the occurring signs.

Recall that a set of weights $\Xi$ is called a chain if $\lambda-\mu \in \Phi$ for all $\lambda, \mu \in \Xi$. Chains of roots correspond to singular subspaces in the geometry of root subgroups; see [23. In the present paper and its sequel devoted to the decomposition of unipotents in adjoint representations, we need sets that consist of two interlaced chains, as shown in Figure 2. Observe that we depict weight diagrams, not weight graphs. The corresponding weight graph is a regular $2 l$-gon where each vertex is joined to all other vertices with the exception of the opposite one.

Such configurations of weights correspond to polar subspaces in the geometry of root subgroups; see [23]. They are also brought forth by the theory of standard monomials in the microweight case [43. Such sets occur so often that they deserve a special name.

Definition. A set of weights $\Omega \subseteq \Lambda(\pi)$ is called a square if it has the following property: $|\Omega| \geq 4$ and for any $\lambda \in \Omega$ the difference $\lambda-\mu$ with all weights $\mu \in \Omega$ except exactly one, denoted by $\bar{\lambda}$, is a root, whereas the difference $\lambda-\bar{\lambda}$ is not a root.

In other words, the distance $d(\lambda, \mu)$ between $\lambda$ and $\mu \in \Omega, \mu \neq \lambda, \bar{\lambda}$, in the weight graph $\mathrm{G}(\Phi, \omega)$ equals 1 . By the condition $|\Omega| \geq 4$, it follows that $d(\lambda, \bar{\lambda})=2$. In other words, $\lambda-\bar{\lambda}$ is the sum of two roots. For instance, when $\pi$ is an adjoint representation or when it is realised as an internal Chevalley module, the angle of $\lambda$ with all other roots $\mu \in \Omega$ except $\bar{\lambda}$, equals $\pi / 3$, whereas $\bar{\lambda}$ is orthogonal to $\lambda$. Obviously, the map $\lambda \mapsto \bar{\lambda}$ is an involution of $\Omega$ without fixed points, so that the order of $\Omega$ is even, $|\Omega|=2 m$ for some $m$.

Consider an arbitrary decomposition of the set $\Omega$ into two disjoint parts $\Omega=\Omega^{+} \amalg \Omega^{-}$, with the property that any two weights of $\Omega^{+}$are adjacent. Then for every $\lambda \in \Omega^{+}$the weight $\bar{\lambda}$ lies in $\Omega^{-}$. Clearly, $\Omega^{-}$is also a chain. For a square of order $2 m$, there are precisely $2^{m}$ ways to decompose it into two disjoint chains. Indeed, for every pair of weights $\lambda, \bar{\lambda}$ exactly one of them belongs to $\Omega^{+}$, and these choices are independent.

Obviously, for the representation $\left(\mathrm{A}_{l}, \varpi_{1}\right)$ there are no squares. This corresponds to the fact that a column of a matrix in $\mathrm{SL}(n, R)$ is not subject to any algebraic equations. For the representation $\left(D_{l}, \varpi_{1}\right)$, whose diagram is reproduced in Figure 2, the whole set of weights forms a square. This means that columns of orthogonal matrices are subject to a single quadratic equation. Our first goal is to describe the maximal squares in other representations. First, we summarize some basic properties of squares. These properties are obvious from Figure 2, but it is exactly what we have to check: all squares have the same structure as the one presented in this figure.

In the following theorems we assume that all roots of $\Phi$ have the same length, and $\pi$ is a microweight or an adjoint representation. Of course, we could easily state similar results for all root systems. However, as we illustrate by the example of $\mathrm{B}_{l}$, our theorems fail to hold as stated. Furthermore, we do not need this for the actual applications we have in mind. For instance, the decomposition of unipotents in vector representations of classical groups can be constructed without any reference to equations whatsoever [45. For other representations of classical groups, it is also easier to give an ad hoc 
construction; see [45, 10]. For microweight representations of $\mathrm{E}_{6}$ and $\mathrm{E}_{7}$ one also can, with a slightly different approach, minimize the reference to equations; see [6, 7, 9, 49]. On the other hand, the group of type $\mathrm{F}_{4}$ is best understood as a twisted form of the group of type $\mathrm{E}_{6}$; see [7, 9]. Thus, intending to use the following results primarily for adjoint representations of types $\mathrm{E}_{6}, \mathrm{E}_{7}, \mathrm{E}_{8}$, we decided not to sacrifice clarity and simplicity for the sake of sham generality.

Theorem 1. Let $\Omega$ be a square. Then the sum $\sigma=\sigma(\Omega)$ of two weights $\lambda+\bar{\lambda}$ at distance 2 does not depend on the choice of $\lambda \in \Omega$. Conversely, a maximal square $\Omega$ is uniquely defined by $\sigma(\Omega)$.

We prove Theorem 1 in two stages. Clearly, in the proof of its first statement it also suffices to consider maximal squares. First, we prove that a maximal square is completely determined by any of its weights $\lambda, \mu$ at distance 2 . In the majority of cases - in all of them? - we consider the following result is contained in the papers by Bruce Cooperstein and Arjeh Cohen on the characterisation of geometries related to parabolic subgroups of Chevalley groups; see in particular 22, 23, and further references in [19] $2^{2}$ In particular, in the most interesting case of adjoint representations of type $\mathrm{E}_{l}$, the answer can be found in 23 , page 357 for $\mathrm{E}_{6}$, page 361 for $\mathrm{E}_{7}$, and page 363 for $\mathrm{E}_{8}$. Nevertheless, we prefer to give a direct combinatorial proof, which does not invoke geometry, since the detailed calculations omitted in 23. play a key role in our construction of elements of root type that stabilize a column.

Theorem 2. Let $d(\lambda, \mu)=2$. Then the set

$$
\Omega=\Omega(\lambda, \mu)=\{\nu \in \Lambda \mid d(\nu, \lambda)=d(\nu, \mu)=1\} \cup\{\lambda, \mu\}
$$

is a maximal square.

Actually, our proof of Theorems 1 and 2 is not a proof, but rather a case-by-case verification. We postpone it to the following sections, and in the meantime state some of their immediate corollaries. Usually, there is a single conjugacy class of pairs $(\lambda, \mu)$ of weights at distance 2 under the action of the Weyl group. The only exceptions occur for $\Phi=\mathrm{B}_{l}, \mathrm{D}_{l}$. In particular, this implies the following corollary.

Corollary 1. Except for the case $\left(\mathrm{D}_{l}, \varpi_{2}\right), l \geq 4$, all maximal squares in $\Lambda^{*}(\pi)$ form a single conjugacy class under the action of the Weyl group.

- In the case of $\left(\mathrm{D}_{l}, \varpi_{2}\right), l \geq 6$, there are two conjugacy classes.

- In the cases of $\left(\mathrm{D}_{4}, \varpi_{2}\right)$ and $\left(\mathrm{D}_{5}, \varpi_{2}\right)$, there are three conjugacy classes.

Theorem 2 allows us to compute the number of squares of a given type: one has to divide the cardinality of the orbit of an ordered pair $(\lambda, \mu)$ of weights at distance 2 by the order of the corresponding square. After that it is very easy to complete the proof of Theorem 1. It suffices to compare the number of squares conjugate to $\Omega$ with the order of the Weyl orbit of $\sigma(\Omega)$. The fact that $\sigma(\Omega)$ is a weight of another representation allows a very transparent parametrization of squares. In particular, among squares of a given type there is exactly one dominant square, corresponding to the dominant weight $\sigma(\Omega)$. This is exactly the senior square constructed in the proof of Theorem 2, starting with the pair consisting of the maximal weight $\omega$ and the maximal among weights at distance 2 from $\omega$.

The following fact plays a crucial role in the construction of a root type element stabilizing a given column, in the decomposition of unipotents.

\footnotetext{
${ }^{2}$ The notation used in those papers translates to our language as follows: singular points $x, y-$ weights $\lambda, \mu ; f(x)$ - weights at distance 1 from $\lambda ; f_{2}(x)$ - weights at distance 2 from $\lambda ;\langle x, y, f(x) \cap f(y)\rangle$ or $S(x, y)-\Omega(\lambda, \mu)$.
} 
Corollary 2. Let $\lambda, \mu \in \Omega, \mu \neq \lambda, \bar{\lambda}$. Then $\lambda-\mu=\bar{\mu}-\bar{\lambda}$. Conversely, if $\lambda_{1}-\mu_{1}=\lambda_{2}-\mu_{2}$ for two distinct pairs of weights $\left(\lambda_{1}, \mu_{1}\right),\left(\lambda_{2}, \mu_{2}\right)$, then $\lambda_{2}=\bar{\mu}_{1}, \mu_{2}=\bar{\lambda}_{1}$.

Let $|\Omega|=2 m$. This corollary shows that the pairwise differences $\lambda-\mu, \lambda, \mu \in \Omega$, take exactly $2 m(m-1)$ distinct values. It is easily seen that the roots $\Delta(\Omega)=\{\lambda-\mu \mid$ $\lambda, \mu \in \Omega\} \subseteq \Phi$ form a subsystem of type $\mathrm{D}_{m}$. Since some systems have few subsystems of type $\mathrm{D}_{m}$, representations of these types do not possess squares of high order. For example, the only squares in representations of type $\mathrm{A}_{l}$ are squares of order 4 when $\Delta(\Omega)=\mathrm{D}_{2} \cong 2 \mathrm{~A}_{1}$, and squares of order 6 when $\Delta(\Omega)=\mathrm{D}_{3} \cong \mathrm{A}_{3}$. The first of these cases represents vanishing of second order minors, while the second represents familiar short Plücker relations of the form $x_{1} x_{-1}-x_{2} x_{-2}+x_{3} x_{-3}=0$, which can be expressed as dependencies among second order minors.

Corollary 3. In every square $\Omega$ there is a unique maximal element $\lambda$ and a unique minimal element $\mu=\bar{\lambda}$. In every square $\Omega$ there is a unique pair of noncomparable elements $\lambda, \bar{\lambda}$.

The following example shows that the assumption that all roots of $\Phi$ have the same length is essential. Theorems 1 and 2 fail for $(\Phi, \pi)=\left(B_{l}, \varpi_{l}\right)$. Indeed, in this case, for $\sigma \in W \varpi_{l-4}$ one has exactly two maximal squares $\Omega_{1}, \Omega_{2}$ of diameter 2 such that $\sigma\left(\Omega_{1}\right)=\sigma\left(\Omega_{2}\right)=\sigma$. Together, these squares form the configuration depicted in Figure 3 .

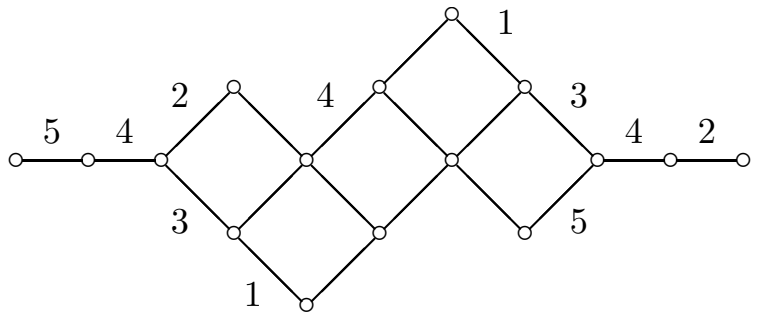

FIgURE 3. $\left(\mathrm{B}_{4}, \bar{\omega}_{4}\right)$

\section{§3. Proof of Theorem 2: Classical cases}

Since in what follows we need a detailed understanding of squares in all representations we consider, we carry out a case-by-case analysis. As we mentioned, in most cases the answer can be extracted from the existing literature. Observe that it suffices to show that, for any two weights $\lambda$ and $\mu$ at distance 2 , the set $\Omega=\Omega_{\lambda \mu}$ consisting of $\lambda, \mu$, and all weights $\nu$ adjacent to both of them, forms a square. This square is automatically maximal and, thus, is completely determined by the weights $\lambda$ and $\mu$. First, we consider microweight representations.

- Vector representations. For vector representations of types $\mathrm{A}_{l}$ and $\mathrm{C}_{l}$, the set of weights is a chain, so that in these cases there are no squares at all. For the type $\mathrm{D}_{l}$, the set of weights is itself a square of order $2 l$. Obviously, the covector representation $\left(A_{l}, \varpi_{l}\right)$ behaves exactly as the vector one.

- Polyvector representations. Now, let $\left(\mathrm{A}_{l}, \varpi_{m}\right), 2 \leq m \leq l-1$, be a polyvector representation distinct from the vector and the covector ones. The weights of this representation are of the form $\lambda=e_{i_{1}}+\cdots+e_{i_{m}}, 1 \leq i_{1}<\cdots<i_{m} \leq l+1$. In other words, they correspond to the $m$-element subsets of $I=\{1, \ldots, l+1\}$, and $\lambda$ corresponds to the subset $X_{\lambda}=\left\{i_{1}, \ldots, i_{m}\right\}$. Recall that the Hamming distance $d_{H}(X, Y)$ between 
two subsets $X$ and $Y$ is the cardinality of their symmetric difference. This is exactly the Hamming distance between their characteristic functions viewed as elements of the vector space $2^{I}$ over $\mathbb{F}_{2}$. Clearly, the Hamming distance between two $m$-element sets is even, and it is easily seen that $d(\lambda, \mu)=d_{H}\left(X_{\lambda}, X_{\mu}\right) / 2$. Thus, two weights $\lambda$ and $\mu$ at distance 2 correspond to subsets $X_{\lambda}, X_{\mu}$ at Hamming distance 4 . Since the Weyl group $W\left(\mathrm{~A}_{l}\right)=S_{l+1}$ transitively acts on $I$, without loss of generality we can assume that $X_{\lambda}=\{1, \ldots, m\}, X_{\mu}=\{1, \ldots, m-2, m+1, m+2\}$ (here we use the fact that $2 \leq m \leq l-1)$. Each set $Y$ at Hamming distance 2 from $X_{\lambda}$ has the form $X_{\lambda} \backslash\{i\} \cup\{j\}$ for some $1 \leq i \leq m, m+1 \leq j \leq l+1$. Similarly, for $Y$ to be at Hamming distance 2 from $X_{\mu}$ it is necessary that $i=m-1, m$ and $j=m+1, m+2$. Indeed, if $1 \leq i \leq m-2$, then $X_{\mu} \backslash Y=\{i, m+1, m+2\}$, so that $d_{H}\left(X_{\mu}, Y\right)=6$. On the other hand, if $m+3 \leq j \leq l+2$, then $Y \backslash X_{\mu}=\{m-1, m, j\}$, and again $d_{H}\left(X_{\mu}, Y\right)=6$. Clearly, this condition is also sufficient, so that there are exactly 4 sets at Hamming distance 2 both from $X_{\lambda}$ and from $X_{\mu}$. Namely, these are $\{1, \ldots, m-1, m+1\},\{1, \ldots, m-2, m, m+1\}$, $\{1, \ldots, m-1, m+2\}$, and $\{1, \ldots, m-2, m, m+2\}$. Clearly, the Hamming distance of any of them to two others equals 2 , and it equals 4 for the remaining subset. Thus, these four sets together with the initial ones form a square of order 6 .

- Half-spin representations. Now we turn to the cases $\left(D_{l}, \varpi_{l}\right),\left(D_{l}, \varpi_{l-1}\right)$ that furnish half-spin representations. The weights of these representations have the form $\frac{1}{2}\left( \pm e_{1} \pm \cdots \pm e_{m}\right)$, with the product of signs being odd in the first case and even in the second. As usual, we identify a weight $\lambda$ with the list of signs $\varepsilon_{1}, \ldots, \varepsilon_{l}$. Now the weights correspond to the subsets of the $l$-element set $\{1, \ldots, l\}$ whose cardinality is even or odd, respectively, depending on the parity of $l$. Namely, to a weight $\lambda$ we assign the set $X$ of indices $i$ such that $\varepsilon_{i}=+1$. Both cases are immediately reduced to the case of all subsets of $\{1, \ldots, l\}$. It suffices to consider their intersection with $\{1, \ldots, l-1\}$; then whether the initial subset contains $l$ is determined by the parity test. Clearly, the distance $d(\lambda, \mu)$ between weights $\lambda$ and $\mu$ equals 1 if the Hamming distance $d_{H}\left(X_{\lambda}, X \mu\right)$ between the corresponding subsets $X_{\lambda}$ and $X_{\mu}$ equals 1 or 2. Similarly, $d(\lambda, \mu)=2$ if $d_{H}\left(X_{\lambda}, X_{\mu}\right)=3$, 4. In general, $d(\lambda, \mu)=-\left[-d_{H}\left(X_{\lambda}, X_{\mu}\right) / 2\right]$, where $[x]$ is the integral part of $x$. Since the Weyl group $W\left(\mathrm{~B}_{l}\right)=$ Oct $_{l}$ acts transitively on $-I \cup I$, without loss of generality we can assume that $X_{\lambda}=I$, and $X_{\mu}=\{1, \ldots, l-3\}$ or $X_{\mu}=\{1, \ldots, l-4\}$. Now, if $X$ is a subset at Hamming distance 1 or 2 both from $X_{\lambda}$ and from $X_{\mu}$, it must contain $X_{\mu}$. In the first/second case it must both contain and avoid at least one/two of the elements $l-2, l-1, l$, or respectively, of the elements $l-3, l-2, l-1, l$. If it does not contain $X_{\mu}$, its Hamming distance from at least one of the sets $X_{\lambda}, X_{\mu}$ would be at least 3. This shows that without loss of generality we can assume that $l=3$ or $l=4$. However, every subset of a three-element set, or respectively, every even subset of a four-element set, is at Hamming distance $\leq 2$ from all subsets of the same type, except its complement. This shows that in each of these cases all $\Omega(\lambda, \mu)$ are squares of order 8 .

- The case $\left(\mathrm{A}_{l}, \mathrm{ad}\right)$. Clearly, $A_{1}^{\perp}=A_{l-2}$. If $\lambda=\delta=e_{1}-e_{l+1}$ is the maximal root, then one can take as $\mu$ the maximal root of $\Delta$, namely, $\mu=e_{2}-e_{l}$. Obviously, the only roots forming angle $\pi / 3$ both with $\lambda$ and with $\mu$, are the mutually orthogonal roots $e_{2}-e_{l+1}$ and $e_{1}-e_{l}$. Clearly, this is a unique square containing $\lambda$ and $\mu$.

- The case $\left(\mathrm{D}_{l}, \mathrm{ad}\right)$. This is the most delicate among all classical cases, because here the orthogonal complement to a root is not irreducible, namely, $\mathrm{A}_{1}^{\perp}=\mathrm{A}_{1}+\mathrm{D}_{l-2}$. If $\lambda=\delta=e_{1}+e_{2}$ is the maximal root, then as $\mu$ we can take either $e_{1}-e_{2}$, or the maximal root $e_{3}+e_{4}$ of $\mathrm{D}_{l-2}$. These two subcases lead to different configurations. In the first case the only roots forming angle $\pi / 3$ both with $\lambda$ and with $\mu$ are the $2(l-2)$ roots $e_{1} \pm e_{i}$, $3 \leq i \leq l$. The remaining $2(l-2)$ roots $e_{2} \pm e_{i}, 3 \leq i \leq l$, forming angle $\pi / 3$ with $\lambda$, form angle $2 \pi / 3$ with $\mu$. Clearly, the differemce of any two roots $e_{1} \pm e_{i}$ and $e_{1} \pm e_{j}$, 
$3 \leq i, j \leq l$, except for $e_{1}+e_{i}$ and $e_{1}-e_{i}$, is again a root. Thus, the set $\Omega(\lambda, \mu)$ forms a square of order $2 l-2$.

In the second subcase the only roots forming angle $\pi / 3$ both with $\lambda$ and with $\mu$ are the following 4 roots: $e_{1}+e_{3}, e_{1}+e_{4}, e_{2}+e_{3}, e_{2}+e_{4}$. The 4 of the remaining roots that form angle $\pi / 3$ with $\lambda$, namely, $e_{1}-e_{3}, e_{1}-e_{4}, e_{2}-e_{3}, e_{2}-e_{4}$, form angle $2 \pi / 3$ with $\mu$, whereas all other $4(l-4)$ roots $e_{1} \pm e_{i}, e_{2} \pm e_{i}, 5 \leq i \leq l$, forming angle $\pi / 3$ with $\lambda$, are orthogonal to $\mu$. Thus, in this case, $\Omega(\lambda, \mu)$ forms a square of order 6 .

The case where $l=4$ is exceptional here. First, we have $2 l-2=6$. Second, $\mathrm{D}_{2}=$ $\mathrm{A}_{1}+\mathrm{A}_{1}$ is reducible, so that the Weyl orbit of squares that corresponds to $\mathrm{D}_{2}$, falls into two orbits. At the same time, these orbits, and the orbit corresponding to $A_{1}$, are fused by outer automorphisms. The exceptional cases $\mathrm{D}_{4}$ and $\mathrm{D}_{5}$ are discussed in more detail at the end of the proof of Theorem 1.

\section{§4. Proof of Theorem 2: Exceptional Cases}

In the present section we finish the proof of Theorem 2. Here, it is much more convenient to use the hyperbolic realization of root systems of type $\mathrm{E}_{l}$, rather than the Euclidean one.

1. Hyperbolic realization of systems $\mathrm{E}_{l}$. We shall use the hyperbolic realization of the root systems of type $E_{l}$ as described in [13. See [8, 12, 26, 48] for a detailed description of this construction. In this realization, $\mathrm{E}_{l}$ is viewed as a subset of the Minkowsky space $V=\mathbb{R}^{l+1}$, with an orthonormal base $e_{0}, e_{1}, \ldots, e_{l}$. Recall that the inner squares are specified as $e_{0}^{2}=-1$ and $e_{i}^{2}=1,1 \leq i \leq l$, while $\left(e_{i}, e_{j}\right)=0, i \neq j$.

We start with the senior case where $l=8$; systems of types $\mathrm{E}_{6}$ and $\mathrm{E}_{7}$ are readily obtained as subsystems of $\mathrm{E}_{8}$. Consider the expansion $v=a e_{0}+b_{1} e_{1}+\cdots+b_{8} e_{8}$ of a vector $v \in V$ with respect to the base $e_{0}, \ldots, e_{8}$, where $a, b_{1}, \ldots, b_{8} \in \mathbb{R}$. Denote by $L$ the lattice in $V$ that consists of all points with integer coordinates, $L=\left\{v \in V \mid a, b_{1}, \ldots, b_{8} \in \mathbb{N}\right\}$. Now we can define $\Phi$ as the subset consisting of all vectors $v \in L$ whose coordinates satisfy the following two equations: $3 a-\left(b_{1}+\cdots+b_{8}\right)=0,-a^{2}+\left(b_{0}^{2}+\cdots+b_{8}^{2}\right)=2$. It is easy to verify that an element of $\Phi$ has one of the following four forms:

- $\beta_{i j}=e_{i}-e_{j}$, where $1 \leq i \neq j \leq 8$;

- $\pm \gamma_{i j h}$, for $\gamma_{i j h}=e_{0}+e_{i}+e_{j}+e_{h}$, where $1 \leq i, j, h \leq 8$ are pairwise distinct;

- $\pm \eta_{i j}$, for $\eta_{i j}=2 e_{0}+e_{1}+\cdots+\widehat{e}_{i}+\cdots+\widehat{e}_{j}+\cdots+e_{8}$, where $1 \leq i \neq j \leq 8$;

- $\pm \zeta_{i}$, for $\zeta_{i}=3 e_{0}+e_{1}+\cdots+2 e_{i}+\cdots+e_{8}$, where $1 \leq i \leq 8$.

As usual, a caret over a summand signifies that this summand should be omitted. Clearly, there are $2\left(\begin{array}{l}8 \\ 2\end{array}\right)=56$ roots of the first type (with $a=0$ ), $2\left(\begin{array}{l}8 \\ 3\end{array}\right)=112$ roots of the second type (with $a= \pm 1$ ), $2\left(\begin{array}{l}8 \\ 2\end{array}\right)=56$ roots of the third type (with $a= \pm 2$ ), and finally, $2\left(\begin{array}{l}8 \\ 1\end{array}\right)=16$ roots of the fourth type (with $a= \pm 3$ ). Thus, the total number of roots equals 240 and it is not hard to check that they form a root system of type $\mathrm{E}_{8}$ in the hyperplane orthogonal to the test vector $3 e_{0}+e_{1}+\cdots+e_{8}$.

As a fundamental system one can take the following eight vectors: $\alpha=e_{2}-e_{1}$, $\alpha=e_{0}+e_{1}+e_{2}+e_{3}, \alpha=e_{i}-e_{i-1}, i=3, \ldots, 8$. Recall that our numbering follows [2], and not [13]. With the above choice, a positive root $\alpha$ has one of the following forms: either $\alpha=e_{i}-e_{0}, 1 \leq i \leq 8$, or $a>0$, i.e., $\alpha=\gamma_{i j h}, \eta_{i j}, \zeta_{i}$. Now, to get a subsystem of type $\mathrm{E}_{7}$, it suffices to take the roots of $\mathrm{E}_{8}$ for which $b_{8}=0$ - or, what is the same, the roots such that $\alpha_{8}$ does not occur in their linear expansion with respect to the fundamental roots. Similarly, to get a subsystem of type $\mathrm{E}_{6}$, it suffices to take the roots of $\mathrm{E}_{8}$ for which $b_{7}=b_{8}=0$ - or, what is the same, the roots such that both $\alpha_{7}$ and $\alpha_{8}$ do not occur in their linear expansion with respect to the fundamental roots. Obviously, $\mathrm{E}_{7}$ does not contain roots of the form $\zeta_{i}$, whereas $\mathrm{E}_{6}$ contains a unique root 
of the form $\eta_{i j}$, namely, $\eta_{78}$, which is the maximal root therein. The maximal roots of $\mathrm{E}_{7}$ and $\mathrm{E}_{8}$ are $\eta_{18}$ and $\zeta_{8}$, respectively.

In what follows we freely invoke data on subsystems of root systems: orthogonal complements, conjugacy under the action of the Weyl group $W=W(\Phi)$, stabilizers in $W$, etc. For the most part, all this can be found already in the classical papers by Borel and de Siebenthal, and by Dynkin; see [12, 26] for further references and a detailed description of the algorithms from those classical papers. In particular, all subsystems of type $\mathrm{A}_{1}$ are conjugate. Recall that, by definition, $\mathrm{A}_{1}$ consists of long roots; a pair of opposite short roots is denoted by $\tilde{\mathrm{A}}_{1}$. The orthogonal complement to $\mathrm{A}_{1}$ is precisely the subsystem in $\Phi$ generated by all fundamental roots except for the fundamental roots adjacent to the negative maximal root in the affine Dynkin diagram. Apart from the case where $\Phi \neq \mathrm{A}_{l}$, the negative maximal root is adjacent to a unique fundamental root. The orthogonal complement of a subsystem $\Delta \subseteq \Phi$ will be denoted by $\Delta^{\perp}$.

By $m \Delta$ one denotes the orthogonal sum of $m$ subsystems isomorphic to $\Delta$. For example, $2 \mathrm{~A}_{1}$ consists of long roots $\pm \alpha$ and $\pm \beta$, where $(\alpha, \beta)=0$. In all cases, apart from $\Phi=\mathrm{B}_{l}, \mathrm{D}_{l}, l \geq 4$, the Weyl group acts transitively on the set of all subsystems of type $2 \mathrm{~A}_{1}$. In the orthogonal case, generally, there are two conjugacy classes of subsystems of type $2 \mathrm{~A}_{1}$, namely, $2 \mathrm{~A}_{1}$ and $\mathrm{D}_{2}$. This is due to the fact that the fundamental root adjacent to the negative maximal root in the affine Dynkin diagram is not terminal. In the standard realization [2, we can take $\left\{ \pm\left(e_{1}-e_{2}\right), \pm\left(e_{3}-e_{4}\right)\right\}$ and $\left\{ \pm\left(e_{1}-e_{2}\right), \pm\left(e_{1}+e_{2}\right)\right\}$ as representatives of $2 \mathrm{~A}_{1}$ and $\mathrm{D}_{2}$, respectively. In the exceptional case $\mathrm{D}_{4}$, another phenomenon is superimposed on top of that. Namely, maximal subsystems of the form $A_{l_{1}}+\cdots+A_{l_{s}}$ with odd $l_{i}$ fall into two conjugacy classes. As a result, in $\mathrm{D}_{4}$ there are three conjugacy classes of subsystems of type $2 \mathrm{~A}_{1}$; namely, the above copy of $2 \mathrm{~A}_{1}$ is not conjugate to $\left\{ \pm\left(e_{1}-e_{2}\right), \pm\left(e_{3}+e_{4}\right)\right\}$.

2. The proof of Theorem 2. To finish the proof of Theorem 2 it only remains to consider six exceptional representations.

- Minimal representations of $\mathrm{E}_{6}$ and $\mathrm{E}_{7}$. These representations are thoroughly studied in $\S 3$ of [47], so that we simply mention that in each of these cases there is a single Weyl orbit of pairs of weights at distance 2 , and for any two such weights $\lambda$ and $\mu$ the set $\Omega(\lambda, \mu)$ forms a square, of order 10 or 12 , respectively. The corresponding geometric objects were studied under different disguises by a number of authors, apparently starting with Freudenthal. For $\mathrm{E}_{6}$ these are the $\alpha_{6}$-objects of the corresponding building, known as symplecta. In the context of the exceptional Jordan algebra they correspond to the inner ideals of rank 1; see references in [25. For $\mathrm{E}_{7}$ these are the $\alpha_{1}$-objects of the corresponding building.

Since the Weyl group $W=W(\Phi)$ transitively acts on roots, without loss of generality we can assume that $\lambda=\delta$ is the maximal root of $\Phi$. Let $\Delta$ be the subsystem orthogonal to $\delta$. If it is irreducible, then the Weyl group $W(\Delta)$ again acts transitively and we can take as $\mu$ any root of $\Delta$, for instance, its maximal root.

- The case $\left(\mathrm{E}_{6}, \mathrm{ad}\right)$. Clearly, $\mathrm{A}_{1}^{\perp}=\mathrm{A}_{5}$. The roots orthogonal to $\delta$ are precisely the roots of the form $\beta_{i j}, 1 \leq i \neq j \leq 6$. Let us fix one of them, say, $\mu=\beta_{61}$, and find all roots that form angle $\pi / 3$ both with $\lambda$ and with $\mu$. Clearly, the roots forming angle $\pi / 3$ with $\lambda=\delta$ are precisely the 20 roots of the form $\gamma_{i j h}, 1 \leq i<j<h \leq 6$. Exactly 6 among them, namely $\gamma_{i j 6}, 2 \leq i<j \leq 5$, form angle $\pi / 3$ with $\mu$. Indeed, 6 of the remaining roots $\gamma_{1 i j}, 2 \leq i<j \leq 5$, form angle $2 \pi / 3$, whereas the 8 remaining roots $\gamma_{i j h}, 2 \leq i<j<h \leq 5$, and $\gamma_{1 i 6}, 2 \leq i \leq 5$, are orthogonal to $\mu$. Clearly, two distinct roots of the form $\gamma_{i j 6}, 2 \leq i<j \leq 5$, and $\gamma_{h k 6}, 2 \leq h<k \leq 5$, form angle $\pi / 3$, with the only exception of $\{i, j, h, k\}=\{1,2,3,4\}$, and the angle $\pi / 2$ in this last case. This means that the set $\Omega(\lambda, \mu)$ forms a square of order 8 . 
- The case $\left(\mathrm{E}_{7}\right.$, ad). Clearly, $\mathrm{A}_{1}^{\perp}=\mathrm{D}_{6}$ and we can take the maximal root $\gamma_{167}$ of that system as $\mu$. Let us find all roots forming angle $\pi / 3$ both with $\lambda$ and with $\mu$. Obviously, the roots forming angle $\pi / 3$ with $\lambda=\delta$ are precisely the following $32=6+20+6$ roots: $\beta_{i 1}, 2 \leq i \leq 7 ; \gamma_{i j h}, 2 \leq i<j<h \leq 7$; and $\eta_{j 8}, 2 \leq i \leq 7$. Among them precisely 8 roots, namely $\gamma_{i 67}, 2 \leq i \leq 5$, and $\eta_{j 8}, 2 \leq j \leq 5$, form angle $\pi / 3$ with $\mu$. Indeed, 8 of the remaining roots $\beta_{h 1}, 2 \leq i \leq 5$, and $\gamma_{i j h}, 2 \leq i<j<h \leq 5$, form angle $2 \pi / 3$, whereas the 16 remaining roots $\beta_{h 1}, \eta_{h 8}, \gamma_{i j h}, 2 \leq i<j \leq 5, h=6,7$, are all orthogonal to $\mu$. Clearly, two distinct roots of the form $\gamma_{i 67}, 2 \leq i \leq 5$, and $\eta_{j 8}, 2 \leq j \leq 5$, form angle $\pi / 3$, except for the case where $i=j$, and angle $\pi / 2$ in this last case. Thus, again the set $\Omega(\lambda, \mu)$ forms a square of order 10 .

- The case $\left(\mathrm{E}_{8}, \mathrm{ad}\right)$. Clearly, $\mathrm{A}_{1}^{\perp}=\mathrm{E}_{7}$ and we can take the maximal root $\eta_{18}$ of this subsystem as $\mu$. Let us find all roots forming angle $\pi / 3$ both with $\lambda$ and with $\mu$. Obviously, the roots forming angle $\pi / 3$ with $\lambda=\delta$ are precisely the following $56=$ $7+21+21+7$ roots: $\beta_{8 i}, 1 \leq i \leq 7 ; \gamma_{i j 8}, 2 \leq i<j \leq 7 ; \eta_{i j}, 2 \leq i<j \leq 7$; and $\zeta_{i}$, $1 \leq i \leq 7$. Exactly 12 among them, the roots $\eta_{1 i}, 2 \leq i \leq 7$, and $\zeta_{j}, 2 \leq j \leq 7$, form angle $\pi / 3$ with $\mu$. Namely, 12 of the remaining roots, $\beta_{8 i}, 2 \leq i \leq 7$, and $\gamma_{1 j 8}, 2 \leq j \leq 7$, form angle $2 \pi / 3$, whereas the remaining 32 roots $\beta_{81}, \zeta_{1}, \gamma_{i j 8}, 2 \leq i<j \leq 7$, and $\eta_{i j}$, $2 \leq i<j \leq 7$, are orthogonal to $\mu$. Clearly, any two roots of the form $\eta_{1 i}, 2 \leq i \leq 7$, and $\zeta_{j}, 2 \leq j \leq 7$, form angle $\pi / 3$, with the exception of the case where $i=j$, and angle $\pi / 2$ in this last case. Thus, also for $\mathrm{E}_{8}$ the set $\Omega(\lambda, \mu)$ forms a square of order 14 .

\section{$\S 5$. Proof of Theorem 1}

In the above proof of Theorem 2, we described the senior square among all maximal squares, in each case. As a matter of fact, this theorem immediately addresses also the problem of their enumeration. Indeed, a maximal square is completely determined by any two of its weights at distance 2. Thus, to find the number of squares of a given type, one has to multiply the number of nonzero weights of the representation $\pi$ by the number of weights at distance 2 from a given weight. For exceptional cases, such as the spin representation of $\mathrm{B}_{l}$ and the adjoint representation of $\mathrm{D}_{l}$, one must take into account the fact that there are more than one orbit of such weights. However, the number of times each square is counted as a result of this process equals its order. Thus, to get the answer one should divide the resulting number by $|\Omega|=2 \mathrm{~m}$.

We summarize the answer in Table 1 . For the cases where $\Phi=\mathrm{D}_{4}, \mathrm{D}_{5}$ some columns of this table need modification; see below. The columns of this table have the following meaning.

- $(\Phi, \omega)$ - a representation.

- $\sigma=\lambda+\bar{\lambda}$ - the sum of two weights at distance 2 in a given square.

- $|\Lambda(\omega)|$ - the number of nonzero weights in the given representation.

- $|\Theta|$ - the number of weights in a given orbit of weights at distance 2 from a given weight. The sum of numbers in this column gives the number of all weights at distance 2 from a fixed nonzero weight.

- $|\Omega|$ — the order of a square corresponding to a given weight.

- $|W(\Phi)|$ - the order of the Weyl group.

- $|W(\Psi)|$ — the order of the stabilizer of $\sigma$ in the Weyl group.

- $N$ - the number of squares of a given type.

Recall that $N=|\Lambda(\omega)| \cdot|\Theta| /|\Omega|$. The proof of Theorem 2 implies that the senior square $\Omega$ of a given type completely determines the weight $\sigma=\sigma(\Omega)$. Since the Weyl group acts both on weights and squares, the same is true for any square. For the converse to hold, 


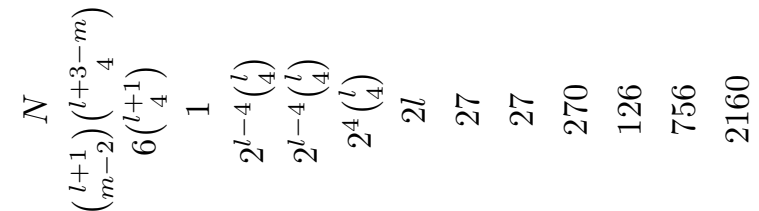

$$
\begin{aligned}
& \ddot{-}
\end{aligned}
$$

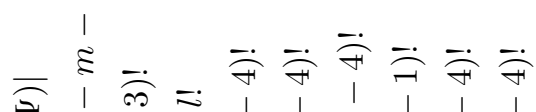

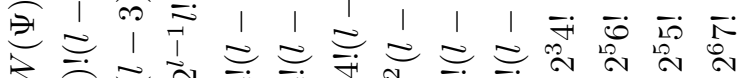

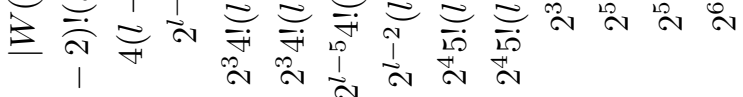

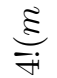

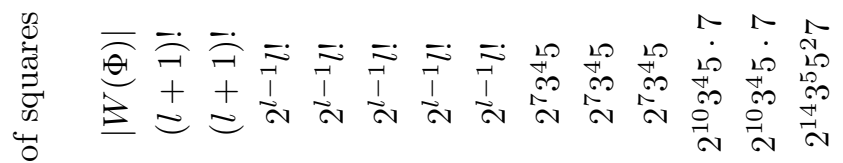

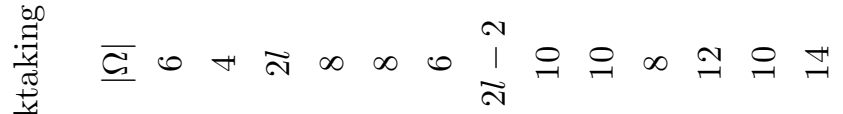

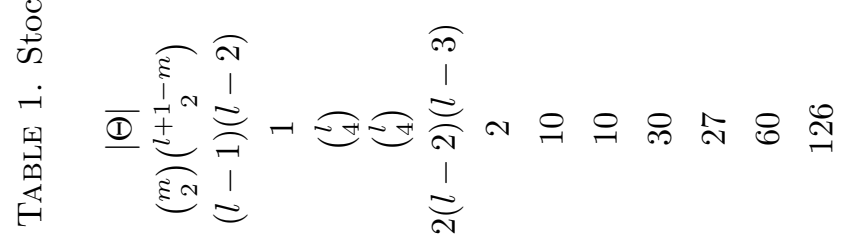

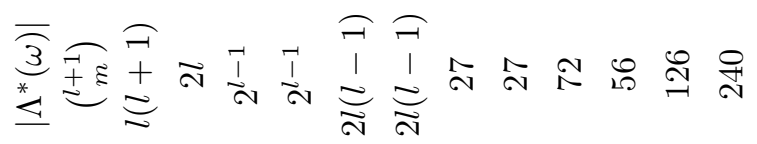

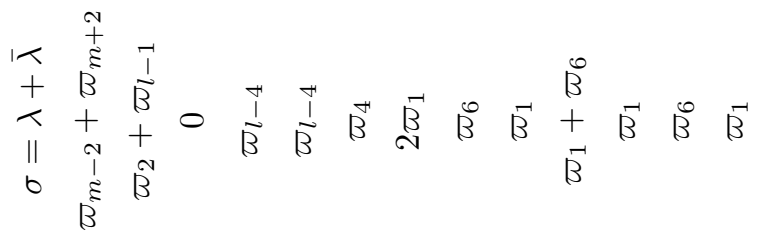

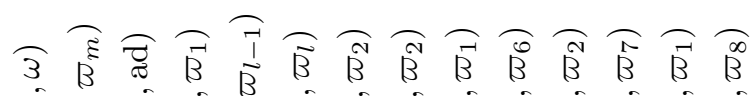

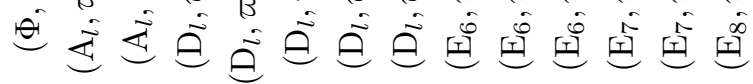


the Weyl orbit of the weight $\sigma$ must have the same order as the Weyl orbit of the square $\Omega$. But the order of the orbit $\sigma$ equals $|W(\Phi) \sigma|=|W(\Phi) / W(\Psi)|$. Summarizing the above, we can infer that the conclusion of Theorem 1 amounts to the identity $N=$ $|W(\Phi) / W(\Psi)|$.

Comparing the values of $N,|W(\Phi)|$, and $|W(\Psi)|$ collected in Table 1, it is easy to observe that such an identity indeed holds in all cases, with the exception of $\Phi=\mathrm{D}_{4}, \mathrm{D}_{5}$. In these cases, some of the columns of Table 1 must be corrected. This proves Theorem 1 in all cases except $\Phi=\mathrm{D}_{4}, \mathrm{D}_{5}$. Let us have a closer look at what happens in the exceptional cases.

- First, whereas for $\Phi=\mathrm{D}_{l}, l \geq 6$, the weight $\varpi_{4}$ equals $e_{1}+e_{2}+e_{3}+e_{4}$, for the exceptional cases $\mathrm{D}_{4}$ and $\mathrm{D}_{5}$ we have $\varpi_{4}=\frac{1}{2}\left(e_{1}+e_{2}+e_{3}+e_{4}\right)$, and thus $\sigma=\varpi_{4}$ should be replaced by $\sigma=2 \varpi_{4}$.

- Second, for these cases the weight $\varpi_{4}$ corresponds to the terminal node of the Dynkin diagram, and thus, the order of its stabilizer should be computed differently and is equal to $|W(\Psi)|=4$ ! for $\Phi=\mathrm{D}_{4}$ and to $|W(\Psi)|=5$ ! for $\Phi=\mathrm{D}_{5}$.

We summarize the answer for the cases $\left(\mathrm{D}_{l}, \varpi_{2}\right), l=4,5$.

- For $\Phi=\mathrm{D}_{4}$, all numbers in the rows corresponding to $\varpi_{4}$ and $2 \varpi_{1}$ coincide. Namely, there are 24 squares of order 6 , which break into 3 series of 8 , with sums $2 \varpi_{1}, 2 \varpi_{3}, 2 \varpi_{4}$, respectively. These squares are fused by outer automorphisms of the root system, but are not conjugate under the action of the Weyl group itself.

- For $\Phi=D_{5}$, the series corresponding to $2 \varpi_{1}$ is preserved, but the series corresponding to $\varpi_{4}$ branches in two, with sums $2 \varpi_{4}$ and $2 \varpi_{5}$, respectively. The latter two series are fused by an outer automorphism, but again they are not conjugate under the action of the Weyl group itself.

We have already mentioned that for $\left(\mathrm{B}_{l}, \varpi_{l}\right), \sigma=\varpi_{l-4}$, the correspondence between the Weyl orbit of $\sigma$ and squares with this sum breaks down. Namely, in this case, $N=2^{l-3}\left(\begin{array}{l}l \\ 4\end{array}\right)$, while $|W(\Phi) / W(\Psi)|=2^{l-4}\left(\begin{array}{l}l \\ 4\end{array}\right)$. This means that for each weight in the orbit of $\sigma$ there are two squares with the sum $\sigma(\Omega)=\sigma$. If $\Omega_{1}$ is one of them, then the other is $\Omega_{2}=w_{l} \Omega_{1}$. For instance, in the case where $\Phi=\mathrm{B}_{4}$, one can take as $\Omega_{1}$ and $\Omega_{2}$ the sets of all weights with an even and with an odd number of pluses, respectively. For these $\Omega_{1}$ and $\Omega_{2}$, the sum $\sigma=\lambda+\bar{\lambda}$ equals

$$
\sigma=(++++)+(----)=(+++-)+(---+)=0 .
$$

It is easy to see them in Figure 3.

\section{§6. EXCEPTiOnAL NUMEROLOGY}

We went to the Summer Garden and started to count trees there. But when the count reached 6, we stopped and began to dispute: some speculated that 7 would follow, and some that 8 .

Daniil Kharms, The sonnet

For the microweight representations of $\mathrm{E}_{6}$ and $\mathrm{E}_{7}$, there are natural a priori correspondences of squares - and thus of equations on the highest weight orbit — with weights of the dual representation or, respectively, with roots of $\mathrm{E}_{7}$. These correspondences can be described as follows; see [47, §3].

- For a weight $\nu \in \Lambda\left(\varpi_{6}\right)$ we set

$$
\Omega(\nu)=\left\{\lambda \in \Lambda\left(\varpi_{1}\right) \mid \nu-\lambda \in \Lambda\left(\varpi_{1}\right)\right\}=\left\{\lambda \in \Lambda\left(\varpi_{1}\right) \mid d(\lambda,-\nu)=2\right\} .
$$


- For a root $\alpha \in \mathrm{E}_{7}$ we set

$$
\Omega(\alpha)=\left\{\lambda \in \Lambda\left(\varpi_{7}\right) \mid \lambda-\alpha \in \Lambda\left(\varpi_{7}\right)\right\} .
$$

In the present section we establish similar correspondences for adjoint representations of Chevalley groups of types $\mathrm{E}_{6}, \mathrm{E}_{7}$ and $\mathrm{E}_{8}$.

- For $\mathrm{E}_{6}$, there is an a priori correspondence of the 270 squares with monomials of the trilinear form $F$ that defines a group of type $\mathrm{E}_{6}$ in the 27-dimensional representation $V\left(\varpi_{1}\right)$. Since this representation is microweight, we have $(\alpha, \lambda)= \pm 1,0$ for any root $\alpha \in \Phi$ and any weight $\lambda \in \Lambda\left(\varpi_{1}\right)$. Clearly, a triad $(\lambda, \mu, \nu)$ determines the square $\Omega=\Omega(\Lambda, \mu, \nu)$ as follows.

Lemma. Assigning to a triad $(\lambda, \mu, \nu)$ the set

$$
\Omega(\lambda, \mu, \nu)=\{\alpha \in \Phi \mid(\alpha, \lambda)=-1,(\alpha, \mu)=0,(\alpha, \nu)=+1\}
$$

we establish an isomorphism of the two following $W\left(\mathrm{E}_{6}\right)$-sets:

- the set of all triads of weights of $V\left(\varpi_{1}\right)$,

- the set of all squares in $\left(\mathrm{E}_{6}, \mathrm{ad}\right)$.

Proof. Indeed, since the Weyl group $W\left(\mathrm{E}_{6}\right)$ acts transitively both on triads and on squares, it suffices to verify that the set $\Omega(\lambda, \mu, \nu)$ is indeed a square for some triad. A straightforward calculation, using the fact that $\lambda=-\varpi_{6}$ and $\mu=\varpi_{1}$, shows that the set $\Omega(\lambda, \mu, \nu)$ corresponding to this particular triad is precisely the senior square $\Omega\left(\delta, \beta_{61}\right)$.

By using exceptional symmetries, similar descriptions can be given also for squares in the adjoint representations of $\mathrm{E}_{7}$ and $\mathrm{E}_{8}$. Namely, scanning Table 1 shows that, occasionally, weights $\sigma$ for squares in different representations coincide, for some special values of parameters $l$ and $m$. This happens, in particular, in the following two cases.

- For $\left(\mathrm{A}_{l}, \varpi_{m}\right)$ and $\left(\mathrm{A}_{l}\right.$, ad $)$ with $l=7, m=4$, the weight equals $\varpi_{2}+\varpi_{6}$.

- For $\left(\mathrm{D}_{l}, \varpi_{l-2}\right)$ and $\left(\mathrm{D}_{l}, \varpi_{2}\right)=\left(\mathrm{D}_{l}\right.$, ad $)$ with $l=8$, the weight equals $\varpi_{4}$.

The only plausible explanation that immediately comes to mind is that this phenomenon should be related to the exceptional embeddings $\mathrm{A}_{7} \subseteq \mathrm{E}_{7}$ and $\mathrm{D}_{8} \subseteq \mathrm{E}_{8}$.

This is indeed the case: each square in $\mathrm{E}_{7}$ can be subdivided into the disjoint union of a square in $\left(A_{7}\right.$, ad $)$ and a square in $\left(A_{7}, \varpi_{4}\right)$, for an appropriate subsystem $A_{7}$. Similarly, each square in $\mathrm{E}_{8}$ can be subdivided into the disjoint union of a square in $\left(\mathrm{D}_{l}\right.$, ad $)$ and a square in $\left(D_{8}, \varpi_{7}\right)$, for an appropriate subsystem $D_{8}$. An evidence to this effect is the coincidence of the entries in the $|\Omega|$ column: $10=4+6$ and $14=6+8$. Obviously, not every square can be obtained in this way for a given subsystem of type $A_{7}$ or $D_{8}$. As a result, it is somewhat harder to verify the coincidence of the entries in the $N$ column; one has to consider all subsystems of type $\mathrm{A}_{7}$ or $\mathrm{D}_{8}$, respectively, and also account for the fact that a square can be subdivided into such parts in a number of different ways.

- For the system $\mathrm{E}_{7}$ we start with the observation that

$$
\left|W\left(\mathrm{E}_{7}\right)\right|=2^{10} \cdot 3^{4} \cdot 5 \cdot 7, \quad\left|W\left(\mathrm{~A}_{7}\right)\right|=8 !=2^{7} \cdot 3^{2} \cdot 5 \cdot 7,
$$

whence $\left|W\left(\mathrm{E}_{7}\right) / W\left(\mathrm{~A}_{7}\right)\right|=2^{3} \cdot 3^{2}=72$.

$\circ$ Since $\left|N_{W\left(\mathrm{E}_{7}\right)}\left(W\left(\mathrm{~A}_{7}\right)\right) / W\left(\mathrm{~A}_{7}\right)\right|=2$, it follows that $\mathrm{E}_{7}$ has exactly 36 subsystems of type $\mathrm{A}_{7}$.

○ The number of squares in a given $A_{7}$ equals $\left(\begin{array}{l}4 \\ 2\end{array}\right)\left(\begin{array}{l}8 \\ 4\end{array}\right)=\left(\begin{array}{l}8 \\ 2\end{array}\right)\left(\begin{array}{l}6 \\ 4\end{array}\right)=420$.

- There are $\left(\begin{array}{l}5 \\ 2\end{array}\right)=10$ ways to subdivide a given square in $\mathrm{E}_{7}$ into a pair of $\mathrm{A}_{7}$-squares. Thus, at first glance, there are $36 \cdot 420 / 10=1512$ squares in $\mathrm{E}_{7}$. However, this is twice the actual number of squares in $\mathrm{E}_{7}$, equal to 756 . What accounts for the mysterious factor of 2 ? 
- For the system $\mathrm{E}_{8}$ we start with the observation that

$$
\left|W\left(\mathrm{E}_{8}\right)\right|=2^{14} \cdot 3^{5} \cdot 5^{2} \cdot 7, \quad\left|W\left(\mathrm{D}_{8}\right)\right|=8 !=2^{7} \cdot 6 !=2^{14} \cdot 3^{2} \cdot 5 \cdot 7,
$$

whence $\left|W\left(\mathrm{E}_{8}\right) / W\left(\mathrm{D}_{8}\right)\right|=3^{3} \cdot 5=135$.

- Since $W\left(\mathrm{D}_{8}\right)$ coincides with its own normalizer in $W\left(\mathrm{E}_{8}\right)$, it follows that $\mathrm{E}_{8}$ has exactly 135 subsystems of type $\mathrm{D}_{8}$.

○ The number of squares in a given $\mathrm{D}_{8}$ equals $2^{4}\left(\begin{array}{l}8 \\ 4\end{array}\right)=1120$.

- There are $\left(\begin{array}{l}7 \\ 3\end{array}\right)=35$ ways to subdivide a given square in $\mathrm{E}_{8}$ into a pair of $\mathrm{D}_{8}$-squares. Thus, at first glance, this should give $135 \cdot 1120 / 35=4320$ squares in $\mathrm{E}_{8}$. Again this is twice the actual number of squares in $\mathrm{E}_{8}$, equal to 2160. Somehow, now we are less surprised.

The extra factor of 2 in the above enumerations can be explained as follows. A closer look inside the squares, which uses the embeddings $2 \mathrm{~A}_{3} \subseteq \mathrm{A}_{7}$ and $2 \mathrm{D}_{4} \subseteq \mathrm{D}_{8}$, shows that a subdivision of a square into two parts is compatible not with every embedding of $2 \mathrm{~A}_{3}$ into $\mathrm{A}_{7}$ or of $2 \mathrm{D}_{4}$ into $\mathrm{D}_{8}$, but precisely with every second such embedding.

However, since in the exceptional numerology 6 is followed by 8 , rather than by 7 , there is a still easier explanation. Indeed, $2160=270 \cdot 8$, whereas 756 is not divisible by 270. It is divisible by 27 though, $756=27 \cdot 28$. We leave further exercises in this style to the amateurs of the Japanese amusements sokoban and sudoku.

\section{$\S 7$. SquARE EQUATIONS}

Kleopatra Platonovna, the features of whom were betraying a deep and awful wavering, approached the wall, tore away a small slab with strange, obscure signs, and threw it to the floor smashing it into smithereens.

Alekse Tolstor̆, Vampire

In the present section we pursue the analysis of equations satisfied by the highest weight orbit. By now we have a good command of their shape. It remains to specify the signs of occurring monomials. In the next section we describe how to find these equations in the weight diagram.

Microweight representations of the Chevalley groups of types $\mathrm{E}_{6}$ and $\mathrm{E}_{7}$ are thoroughly examined in [47. Here we limit ourselves to the adjoint representation $\pi=$ ad. As before, we assume that $\Phi$ is simply-laced. In this case the set $\Lambda^{*}(\pi)$ of nonzero weights of $\pi$ is the root system $\Phi$ itself. Now, to each square $\Omega \subseteq \Lambda^{*}(\pi)$ we assign a certain sign matrix $c=c_{\Omega}$ of degree $|\Omega|$. Under our assumption on $\Phi$ all entries of the sign matrix $c$ are indeed equal to \pm 1 .

Definition. The sign matrix $c=c_{\Omega}$, where $\Omega$ is a square, is defined as follows. Its columns and rows are indexed by weights in $\Omega$. Its entries are specified by

$$
c(\alpha, \beta)=c_{\Omega}(\alpha, \beta)=\left\{\begin{array}{lll}
1 & \text { if } & \alpha=\beta, \bar{\beta} \\
-N_{\alpha,-\beta} N_{\bar{\alpha},-\bar{\beta}} & \text { if } & \alpha \neq \beta, \bar{\beta} .
\end{array}\right.
$$

Let $|\Omega|=2 m$. It is natural to order $\Omega$ as follows: if a root $\alpha \in \Omega$ stands in the $i$ th position, the root $\bar{\alpha}$ stands in the $(2 m+1-i)$ th position. By definition, $c(\alpha, \beta)=c(\bar{\alpha}, \bar{\beta})$.

Now we are all set to prove the following analog of Theorem 3 in [47]. It plays a crucial role both in the construction of unipotent elements stabilizing a column of a matrix in a Chevalley group, in the adjoint representation, and in the proof of decomposition of unipotents in this case. However, it turns out that its proof is a straightforward generalization of Lemma 10 in 47 . 
Theorem 3. Let $c=c_{\Omega}$ be the sign matrix corresponding to a given square $\Omega$. Then $c$ has the following properties.

- It is symmetric: $c(\alpha, \beta)=c(\beta, \alpha)$.

- It is symmetric with respect to the reflections North-South and West-East:

$$
c(\alpha, \beta)=c(\bar{\alpha}, \beta), \quad c(\alpha, \beta)=c(\alpha, \bar{\beta}) .
$$

- It is multiplicative: $c(\alpha, \beta) c(\beta, \gamma)=c(\alpha, \gamma)$.

Proof. If there are exactly two distinct roots among $\alpha, \bar{\alpha}, \beta, \bar{\beta}$, then the first and the second properties are obvious; all occurring entries are equal to +1 . Thus, let $\alpha \neq \beta, \bar{\beta}$. The first property immediately follows from identity (C1) for structure constants 3 In its turn, the second property is a variation of the 2 -cocycle equation. Indeed, $\alpha-\beta=\bar{\beta}-\bar{\alpha}$, and applying identity (C5) to the relation $\alpha-\beta+\alpha-\beta=0$, we get

$$
N_{\alpha,-\beta} N_{\bar{\alpha},-\bar{\beta}}+N_{-\beta, \bar{\alpha}} N_{a,-\bar{\beta}}+N_{\bar{\alpha}, \alpha} N_{-\beta,-\bar{\beta}}=0 .
$$

The last summand equals 0 . Applying to the second summand identity $(\mathrm{C} 2)$ and carrying it over to the right-hand side, we get the desired identities.

Obviously, the third property is the most interesting one. If there are exactly four distinct roots among $\alpha, \bar{\alpha}, \beta, \bar{\beta}, \gamma, \bar{\gamma}$, then there is nothing to prove. Indeed, in this case at least one of the entries $c(\alpha, \beta), c(\beta, \gamma), c(\alpha, \gamma)$ equals +1 by the first two properties. Thus, without loss of generality, we can assume that all six $\operatorname{roots} \alpha, \bar{\alpha}, \beta, \bar{\beta}, \gamma, \bar{\gamma}$ are distinct. By definition,

$$
x=c(\alpha, \beta) c(\beta, \gamma)=N_{\alpha,-\beta} N_{\bar{a},-\bar{\beta}} N_{\beta,-\gamma} N_{\bar{\beta},-\bar{\gamma}} .
$$

Applying identity (C1), we get $x=N_{\beta,-\alpha} N_{\beta,-\gamma} N_{\bar{\beta},-\bar{\alpha}} N_{\bar{\beta},-\bar{\gamma}}$. Now, applying identity (C2) to the relations $\beta-\alpha+(\alpha-\beta)=0, \beta-\gamma+(\gamma-\beta)=0$ and similar relations with $\alpha, \beta, \gamma$ replaced by $\bar{\alpha}, \bar{\beta}, \bar{\gamma}$, we get

$$
x=\left(N_{-\alpha, \alpha-\beta} N_{-\gamma, \gamma-\beta}\right)\left(N_{-\bar{\alpha}, \bar{\alpha}-\bar{\beta}} N_{-\bar{\gamma}, \bar{\gamma}-\bar{\beta}}\right) .
$$

Again by identity $(\mathrm{C} 1)$, we conclude that

$$
x=\left(N_{-\alpha, \alpha-\beta} N_{\beta-\gamma, \gamma}\right)\left(N_{-\bar{\alpha}, \bar{\alpha}-\bar{\beta}} N_{\bar{\beta}-\bar{\gamma}, \bar{\gamma}}\right) .
$$

Applying identity $(\mathrm{C} 3)$ to the relations $-\alpha+(\alpha-\beta)+(\beta-\gamma)+\gamma=0$ and $-\bar{\alpha}+$ $(\bar{\alpha}-\bar{\beta})+(\bar{\beta}-\bar{\gamma})+\bar{\gamma}=0$, and using the fact that one of the summands equals 0 (for example, $\left.N_{\beta-\gamma,-\alpha}=N_{\bar{\beta}-\bar{\gamma},-\bar{\alpha}}=0\right)$, we see that

$$
x=\left(N_{a-\beta, \beta-\gamma} N_{-\alpha, \gamma}\right)\left(N_{\bar{\alpha}-\bar{\beta}, \bar{\beta}-\bar{\gamma}} N_{-\bar{\alpha}, \bar{\gamma}}\right) .
$$

Using relations $\alpha-\beta=\bar{\beta}-\bar{\alpha}$ and $\gamma-\beta=\bar{\beta}-\bar{\gamma}$ and applying identity (C1), we can conclude that

$$
x=N_{-\alpha, \gamma} N_{-\bar{\alpha}, \bar{\gamma}}=N_{a,-\gamma} N_{\bar{\alpha},-\bar{\gamma}}=c(\alpha, \gamma) .
$$

Now we are all set to define the columns subject to the equations from the Weyl orbit of the senior equation. Unfortunately, for adjoint representations the above equations (by far!) do not exhaust all equations satisfied by the highest weight orbit. For example, none of these equations involves zero-weight coordinates.

Definition. We say that a vector $a=\left(a_{\lambda}\right) \in V, \lambda \in \Lambda(\pi)$, is luminous if for each square $\Omega \subseteq \Lambda^{*}(\pi)$ we have

$$
\sum c_{\Omega}(\beta, \alpha) a_{\alpha} a_{\bar{\alpha}}=0
$$

where the sum is taken over all weights $\alpha$ belonging to a maximal chain $\Omega^{+} \subseteq \Omega$, whereas $\beta \in \Omega$ is any fixed weight.

\footnotetext{
${ }^{3}$ Here and below, the numbering of identities satisfied by structure constants refers to 4 .
} 
Observe that these equations are indeed well defined, and depend on the square $\Omega$ alone, not on the chain $\Omega^{+} \subseteq \Omega$ and the weight $\beta \in \Omega$. As a matter of fact, it is obvious that they involve the same monomials, and it only remains to verify that the signs comply. But this is exactly what we know from Theorem 3 . The property $c(\beta, \alpha)=c(\beta, \bar{\alpha})$ shows that the sign does not depend on the choice of a chain. On the other hand, the multiplicativity of $c(\beta, \alpha)$ implies that $\sum c(\gamma, \alpha) a_{\alpha} a_{\bar{\alpha}}=c(\gamma, \beta) \sum c(\beta, \alpha) a_{\alpha} a_{\bar{\alpha}}$, so that the resulting equation does not depend on the choice of $\beta$.

\section{$\S 8$. Square Equations in The Diagram}

Thus, the resulting equations have the form

$$
a_{\gamma_{1}} a_{\gamma_{-1}} \pm a_{\gamma_{2}} a_{\gamma_{-2}} \pm \cdots \pm a_{\gamma_{m}} a_{\gamma_{-m}}=0 .
$$

Below we briefly describe how to find these equations in the diagram and what they look like in the most familiar cases 4 Corollary 3 asserts that in each square there is exactly one pair of noncomparable weights. Thus, the square equations do correspond to squares visible in the weight diagram. Actually, some of these squares are depicted as quadrangles built up of several small squares. For instance, in Figure 3 there are 10 squares, and in Figure 1 there are 27 of them.

Diagrams of the vector representations $\left(\mathrm{A}_{l}, \varpi_{1}\right)$ and $\left(\mathrm{C}_{l}, \varpi_{1}\right)$ are chains and do not have squares at all. This means that there are no equations whatsoever on the highest weight orbit in these representations. Over an arbitrary field every unimodular column can be completed to a unimodular matrix, and every unimodular column of even height can be completed to a symplectic matrix 5 The same is true for the covector representation $\left(A_{l}, \varpi_{l}\right)$, whose diagram is obtained from the diagram of the vector representation $\left(A_{l}, \varpi_{1}\right)$ by inverting the positive direction. For the vector representation of the even orthogonal group $\left(\mathrm{D}_{l}, \varpi_{1}\right)$ this is not the case. This diagram forms a square itself, and indeed, to be the first column of an orthogonal matrix, a unimodular column has to lie on some quadric, in other words, it has to satisfy one quadratic equation.

In polyvector representations $\left(\mathrm{A}_{l}, \varpi_{m}\right), 2 \leq m \leq l-1$, there are a number of squares of order 6 , which beget equations of the form $x_{i j} x_{h k}-x_{i h} x_{j k}+x_{i k} x_{j h}=0$, the celebrated Plücker relations. When we realize a polyvector representation as an exterior power of the vector representation, these relations are interpreted as relations among minors all of whose columns, except two, coincide. It is well known, that these relations are not independent. In the weight diagram, this is expressed by the fact that a square can consist of several small squares. As a first approximation, the number of independent equations is equal to the number of small squares. Actually, this estimate is still too large. Say, in Figure 6 in [42, which corresponds to the case of $\left(A_{5}, \varpi_{3}\right)$, we see 12 small squares, whereas the actual dimension of the highest weight orbit is 10 , and not 8 . This is due to a higher order relation among equations, represented by the cube: only 4 out of 6 small squares constituting this cube are independent. In general, new higher order relations can occur in each degree, represented by hypercubes.

In the weight diagrams of spin and half-spin representations $\left(\mathrm{B}_{l}, \varpi_{l}\right),\left(\mathrm{D}_{l}, \varpi_{l-1}\right)$, $\left(\mathrm{D}_{l}, \varpi_{l}\right)$ (compare, e.g., Figure 3 ), there are plenty of squares of order 8 . These squares beget equations of the form

$$
x_{++++} x_{----}-x_{++--} x_{--++}+x_{+-+-} x_{-+-+}-x_{+--+} x_{-++-}=0,
$$

the celebrated Cartan equations, which define pure spinors. For instance, in Figure 3 we see 10 such equations, of which 5 are independent.

\footnotetext{
${ }^{4}$ In algebraic geometry one usually considers not the highest weight orbit itself, but rather the corresponding projective variety. For the cases we consider these are certain flag varieties.

${ }^{5}$ Of course, over a ring this is not the case in general, since not every projective module is free.
} 
The cases of $\left(\mathrm{E}_{6}, \varpi_{1}\right),\left(\mathrm{E}_{6}, \varpi_{6}\right)$, and $\left(\mathrm{E}_{7}, \varpi_{7}\right)$, were discussed in [4]. For example, for $\left(E_{6}, \varpi_{1}\right)$ in Figure 1 we see 27 equations, of which 10 are independent. In [47] we explained how to read the signs of monomials in these equations directly in the diagram. These 27 quadratic equations determine a 16-dimensional projective variety, known as the projective octave plane; see the references in [25, 28, 40. These equations express the vanishing of all 27 first order partial derivatives of an invariant cubic form in 27 variables. A complete list of the 27 quadratic equations is reproduced in [21, [8]. For $\mathrm{E}_{7}$ there are 126 equations, of which 28 are independent. The list of 28 independent equations up to signs can be found, for example, in [41].

For the adjoint representation $\left(\mathrm{A}_{l}, \mathrm{ad}\right)$ all squares have order 4 . The corresponding equations have the form $x_{i h} x_{j k}-x_{i k} x_{j h}=0$ and express the following simple fact of life: in a matrix of rank 1 all second order minors avoiding the principal diagonal are equal to 0 . Obviously, for adjoint representations one has to consider also another type of equations, involving zero weight coordinates. We do not discuss these equations in the present paper 6 Once again, equations for the adjoint representation $\left(D_{l}, \varpi_{2}\right)$ are nothing else than Plücker relations for $\left(\mathrm{A}_{l-1}, \varpi_{2}\right)$.

As we have seen, squares in the adjoint representations $\left(\mathrm{E}_{l}\right.$, ad) have orders 8,10 , and 14 , respectively. As a result, the corresponding equations are slightly more complicated than in the preceding cases, The diagrams of these representations are depicted in [46, 42] and in [4]. In fact, a full-scale analysis of these cases requires a more detailed study of the geometry of orbits. However, already these equations afford construction of a root type element stabilizing all coordinates of a column, except maybe the coordinates indexed by a single square, say, the senior one.

In [5] the author intends to apply these results to construct decomposition of unipotents in the adjoint representation of the Chevalley group of type $\mathrm{E}_{6}$.

\section{REFERENCES}

[1] A. Borel, Properties and linear representations of Chevalley groups, Seminar on Algebraic Groups and Related Finite Groups (Inst. Adv. Study, Princeton, NJ, 1968/1969), Lecture Notes in Math., vol. 131, Springer-Verlag, Berlin-New York, 1970, pp. 1-55. MR0258838 (41:3484)

[2] N. Bourbaki, Lie groups and Lie algebras. Ch. 4-6, Springer-Verlag, Berlin, 2002. MR1890629 (2003a:17001)

[3] , Lie groups and Lie algebras. Ch. 7-9, Springer-Verlag, Berlin, 2005. MR2109105 (2005h:17001)

[4] N. A. Vavilov, Can one see the signs of structure constants? Algebra i Analiz 19 (2007), no. 4, 34-68; English transl., St. Petersburg Math. J. 19 (2008), no. 4, 519-543. MR.2381932 (2009b:20087)

[5] Decomposition of unipotents in adjoint representation of the Chevalley group of type $\mathrm{E}_{6}$, Algebra i Analiz (to appear). (Russian)

[6] N. A. Vavilov and M. R. Gavrilovich, An $\mathrm{A}_{2}$-proof of structure theorems for Chevalley groups of types $\mathrm{E}_{6}$ and $\mathrm{E}_{7}$, Algebra i Analiz 16 (2004), no. 4, 54-87; English transl., St. Petersburg Math. J. 16 (2005), no. 4, 649-672. MR2090851 (2005m:20115)

[7] N. A. Vavilov, M. R. Gavrilovich, and S. I. Nikolenko, The structure of Chevalley groups: A proof from the Book, Zap. Nauchn. Sem. S.-Peterburg. Otdel. Mat. Inst. Steklov. (POMI) 330 (2006), 36-76; English transl., J. Math. Sci. (N.Y.) 140 (2007), no. 5, 626-645. MR.2253566 (2007k:20108)

[8] N. A. Vavilov, A. Yu. Luzgarev, and I. M. Pevzner, The Chevalley group of type $\mathrm{E}_{6}$ in the 27dimensional representation, Zap. Nauchn. Sem. S.-Peterburg. Otdel. Mat. Inst. Steklov. (POMI) 338 (2006), 5-68; English transl., J. Math. Sci. (N.Y.) 145 (2007), no. 1, 4697-4736. MR2354606 (2009b:20022)

${ }^{6}$ Perhaps, it is more advantageous to consider reducible representations and to choose a base of the zero subspace indexed by some weights in the Weyl orbit of the corresponding fundamental weight. For instance, in the restriction of the adjoint representation of $\operatorname{GL}(l+1, R)$ to $\operatorname{SL}(l+1, R)$, one can choose a base of the zero subspace, indexed by the weights $\widehat{e}_{1}, \ldots, \widehat{e}_{l+1}$. Then the above equations also apply to zero weights and are the only equations contributing to the stabilization of columns. 
[9] N. A. Vavilov and S. I. Nikolenko, A2-proof of structure theorems for Chevalley groups of type $\mathrm{F}_{4}$, Algebra i Analiz 20 (2008), no. 4, 27-63; English transl. in St. Petersburg Math. J. 20 (2009), no. 4. MR2473743

[10] N. A. Vavilov and E. Ya. Perel'man, Polyvector representations of $\mathrm{GL}_{n}$, Zap. Nauchn. Sem. S.Peterburg. Otdel. Mat. Inst. Steklov. (POMI) 338 (2006), 69-97; English transl., J. Math. Sci. (N.Y.) 145 (2007), no. 1, 4737-4750. MR2354607 (2009c:20083)

[11] N. A. Vavilov, E. B. Plotkin, and A. B. Stepanov, Calculations in Chevalley groups over commutative rings, Dokl. Akad. Nauk SSSR 307 (1989), no. 4, 788-791; English transl., Soviet Math. Dokl. 40 (1990), no. 1, 145-147. MR1020667 (90j:20093)

[12] N. A. Vavilov and N. P. Kharchev, Orbits of the stabilizer of subsystems, Zap. Nauchn. Sem. S.Peterburg. Otdel. Mat. Inst. Steklov. (POMI) 338 (2006), 98-124; English transl., J. Math. Sci. (N.Y.) 145 (2007), no. 1, 4751-4764. MR2354608

[13] Yu. I. Manin, Cubic forms: Algebra, geometry, arithmetic, Nauka, Moscow, 1972; English transl., North-Holland Math. Library, vol. 4, North-Holland Publ. Co., Amsterdam-London, 1974. MR 0360592(50:13040) MR0460349 (57:343)

[14] T. A. Springer, Linear algebraic groups, Itogi Nauki i Tekhniki Sovrem. Probl. Mat. Fundam. Napravleniya, vol. 55, Algebraic Geometry IV, VINITI, Moscow, 1989, pp. 5-136; English transl., Encyclopaedia Math. Sci., vol. 55, Springer-Verlag, Berlin, 1994, pp. 1-121. MR1100484 (92g:20061) MR:1309681 (95g:14002)

[15] R. Steinberg, Lectures on Chevalley groups, Yale Univ., New Haven, Conn., 1968. MR0466335 $(57: 6215)$

[16] M. Aschbacher, The 27-dimensional module for $\mathrm{E}_{6}$. I-IV, Invent. Math. 89 (1987), no. 1, 159-195; J. London Math. Soc. 37 (1988), 275-293; Trans. Amer. Math. Soc. 321 (1990), 45-84; J. Algebra 131 (1990), 23-39. MR0892190||(88h:20045) MR0928524|(89a:20041) MR0986684||(90m:20044) MR 1054997 (91f:20049)

[17] , Some multilinear forms with large isometry groups, Geom. Dedicata 25 (1988), no. 1-3, 417-465. MR0925846 (89c:20067)

[18] R. W. Carter, Simple groups of Lie type, 2nd ed., Wiley, New York, 1989. MR1013112 (90g:20001)

[19] A. M. Cohen and B. N. Cooperstein, A characterization of some geometries of Lie type, Geom. Dedicata 15 (1983), no. 1, 73-105. MR0732544 (85c:51010)

[20] $\longrightarrow$ The 2-spaces of the standard $\mathrm{E}_{6}(q)$-module, Geom. Dedicata 25 (1988), no. 1-3, 467-480. MR 0925847 (89c:51013)

[21] A. M. Cohen and R. H. Cushman, Gröbner bases and standard monomial theory, Computational Algebraic Geometry (Nice, 1992), Progr. Math., vol. 109, Birkhäuser Boston, Boston, MA, 1993, pp. 41-60. MR.1230857 (94g:13017)

[22] B. N. Cooperstein, A characterization of some Lie incidence structures, Geom. Dedicata 6 (1977), no. 2, 205-258. MR0461279 (57:1264)

[23] - The geometry of root subgroups in exceptional groups. I, II, Geom. Dedicata 8 (1979), no. 3, 317-381; 15 (1983), no. 1, 1-45. MR0550374 (81e:20022) MR0732540 (85h:20019)

[24] The fifty-six-dimensional module for $\mathrm{E}_{7}$. I. A four form for $\mathrm{E}_{7}$, J. Algebra 173 (1995), 361-389. MR1325780 (96f:20063)

[25] J. R. Faulkner and J. C. Ferrar, Exceptional Lie algebras and related algebraic and geometric structures, Bull. London Math. Soc. 9 (1977), 1-35. MR0444729 (56:3079)

[26] A. L. Harebov and N. A. Vavilov, On the lattice of subgroups of Chevalley groups containing a split maximal torus, Comm. Algebra 24 (1996), no. 1, 109-133. MR.1370526 (97a:20077)

[27] S. J. Haris, Some irreducible representations of exceptional algebraic groups, Amer. J. Math. 93 (1971), no. 1, 75-106. MR0279103 (43:4829)

[28] A. Iliev and L. Manivel, On the Chow ring of the Cayley plane, Compositio Math. 141 (2005), 146-160. MR2099773 (2005h:14008)

[29] H. Kaji and O. Yasukura, Projective geometry of Freudenthal's varieties of certain type, Michigan Math. J. 52 (2004), no. 3, 515-542. MR2097396 (2005i:14069)

[30] V. Lakshmibai, C. Musili, and C. S. Seshadri, Geometry of $G / P$. III. Standard monomial theory for a quasi-minuscule P, Proc. Indian Acad. Sci. Sect. A 88 (1979), no. 3, 93-177. MR0561813 (81g:14023c)

[31] , Geometry of G/P. IV. Standard monomial theory for classical types, Proc. Indian Acad. Sci. Sect. A 88 (1979), no. 4, 279-362. MR0553746 (81g:14023d)

[32] V. Lakshmibai and C. S. Seshadri, Geometry of G/P. II. The work of de Concini and Procesi and the basic conjectures, Proc. Indian Acad. Sci. Sect. A 87 (1978), no. 2, 1-54. MR0490244 (81g:14023b)

[33] , Geometry of G/P. V, J. Algebra 100 (1986), 462-557. MR0840589 (87k:14059) 
[34] - Standard monomial theory, Hyderabad Conf. on Algebraic Groups (Hyderabad, 1989), Manoj Prakashan, Madras, 1991, pp. 279-322. MR1131317 (92k:14053)

[35] V. Lakshmibai and J. Weyman, Multiplicities of points on a Schubert variety in a minuscule $G / P$, Adv. Math. 84 (1990), 179-208. MR.1080976 (92a:14058)

[36] W. Lichtenstein, A system of quadrics describing the orbit of the highest weight vector, Proc. Amer. Math. Soc. 84 (1982), no. 4, 605-608. MR0643758 (83h:14046)

[37] H. Matsumoto, Sur les sous-groupes arithmétiques des groupes semi-simples déployés, Ann. Sci. École Norm. Sup. (4) 2 (1969), 1-62. MR0240214 (39:1566)

[38] S. Nikolenko and N. Semenov, Chow ring structure made simple, arXiv:math.AG/0606335 (2006), $1-17$.

[39] D. I. Panyushev, The actions of a maximal torus on orbits of highest weight vectors, J. Algebra 212 (1999), no. 2, 683-702. MR.1676860 (2000f:14073)

[40] V. Petrov, N. Semenov, and K. Zainoulline, Zero cycles on a twisted Cayley plane, arXiv:math.AG/ 0508200v2 (2005), 1-15; Canad. Math. Bull. 51 (2008), no. 1, 114-124. MR2384744 (2008m:20076)

[41] E. B. Plotkin, On the stability of the $\mathrm{K}_{1}$-functor for Chevalley groups of type $\mathrm{E}_{7}$, J. Algebra 210 (1998), 67-85. MR 1656415 (99k:20099)

[42] E. Plotkin, A. Semenov, and N. Vavilov, Visual basic representations: An atlas, Internat. J. Algebra Comput. 8 (1998), no. 1, 61-95. MR.1492062 (98m:17010)

[43] C. S. Seshadri, Geometry of G/P. I. Theory of standard monomials for minuscule representations, C. P. Ramanujam - a Tribute, Tata Inst. Fund. Res. Stud. in Math., vol. 8, Springer, Berlin-New York, 1978, pp. 207-239. MR0541023 (81g:14023a)

[44] M. R. Stein, Stability theorems for $\mathrm{K}_{1}, \mathrm{~K}_{2}$ and related functors modeled on Chevalley groups, Japan. J. Math. (N.S.) 4 (1978), no. 1, 77-108. MR0528869 (81c:20031)

[45] A. V. Stepanov and N. A. Vavilov, Decomposition of transvections: A theme with variations, $K-$ Theory 19 (2000), 109-153. MR1740757(2000m:20076)

[46] N. Vavilov, Structure of Chevalley groups over commutative rings, Nonassociative Algebras and Related Topics (Hiroshima, 1990), World Sci. Publ., River Edge, NJ, 1991, pp. 219-335. MR1150262 (92k:20090)

[47] , A third look at weight diagrams, Rend. Sem. Mat. Univ. Padova 104 (2000), no. 1, 201-250. MR 1809357 (2001i:20099)

[48] _ Do it yourself structure constants for Lie algebras of types $\mathrm{E}_{l}$, Zap. Nauchn. Sem. S.Peterburg. Otdel. Mat. Inst. Steklov. (POMI) 281 (2001), 60-104; English transl., J. Math. Sci. (N.Y.) 120 (2004), no. 4, 1513-1548. MR1875718 (2002k:17022)

[49] _ An $\mathrm{A}_{3}$-proof of structure theorems for Chevalley groups of types $\mathrm{E}_{6}$ and $\mathrm{E}_{7}$, Internat. J. Algebra Comput. 17 (2007), no. 5-6, 1283-1298. MR2355697

[50] N. Vavilov and E. Plotkin, Chevalley groups over commutative rings. I. Elementary calculations, Acta Appl. Math. 45 (1996), no. 1, 73-113. MR.1409655 (97h:20056)

Department of Mathematics and Mechanics, St. Petersburg State University, UniversitetSkil Pr. 28, Petrodvorets, 198504 St. Petersburg, Russia

E-mail address: nikolai-vavilov@yandex.ru

Received 1/APR/2007

Translated by THE AUTHOR 